Large enhancement of magnetocaloric and barocaloric effect by hydrostatic pressure in $\mathrm{La}\left(\mathrm{Fe}_{0.92} \mathrm{Co0.08}\right)_{11.9} \mathrm{Si}_{1.1}$ with $\mathrm{NaZn} 13$-type structure

Jiazheng Hao, Fengxia Hu, ${ }^{*}$ Jian-Tao Wang, Fei-Ran Shen, Zibing Yu, Houbo Zhou, Hui Wu, Qingzhen Huang, Kaiming Qiao, Jing Wang, Jun He, Lunhua He, Ji-Rong Sun, and Baogen Shen*

Series title:

I. The neutron powder diffraction (NPD) pattern collected at $300 \mathrm{~K}$ in the absence of pressure for $\mathrm{La}\left(\mathrm{Fe}_{0.92} \mathrm{Co0} .08\right){ }_{11.9} \mathrm{Si}_{1.1}$ compound.

II. Crystal structure parameters of $\mathrm{La}\left(\mathrm{Fe}_{0.92} \mathrm{Co} \mathrm{Co}_{0.08}\right)_{11.9} \mathrm{Si}_{1.1}$ compound under different pressures obtained from the refinements of power neutron diffraction data.

III. The total and partial electronic DOS of up spins and down spins for $\mathrm{La}(\mathrm{Fe} 0.92 \mathrm{Co0} .08){ }_{11.9} \mathrm{Si}_{1.1}$ compound in the ferromagnetic state at ambient pressure.

IV. Evaluation of the enlarged $\Delta V / V$ to the enhancement of $\mathrm{BCE}$ by hydrostatic pressure. 
I. The neutron powder diffraction (NPD) pattern collected at $300 \mathrm{~K}$ in the absence of pressure for $\operatorname{La}\left(\mathrm{Fe}_{0.92} \mathrm{Co0} .08\right){ }_{11.9} \mathrm{Si}_{1.1}$ compound.

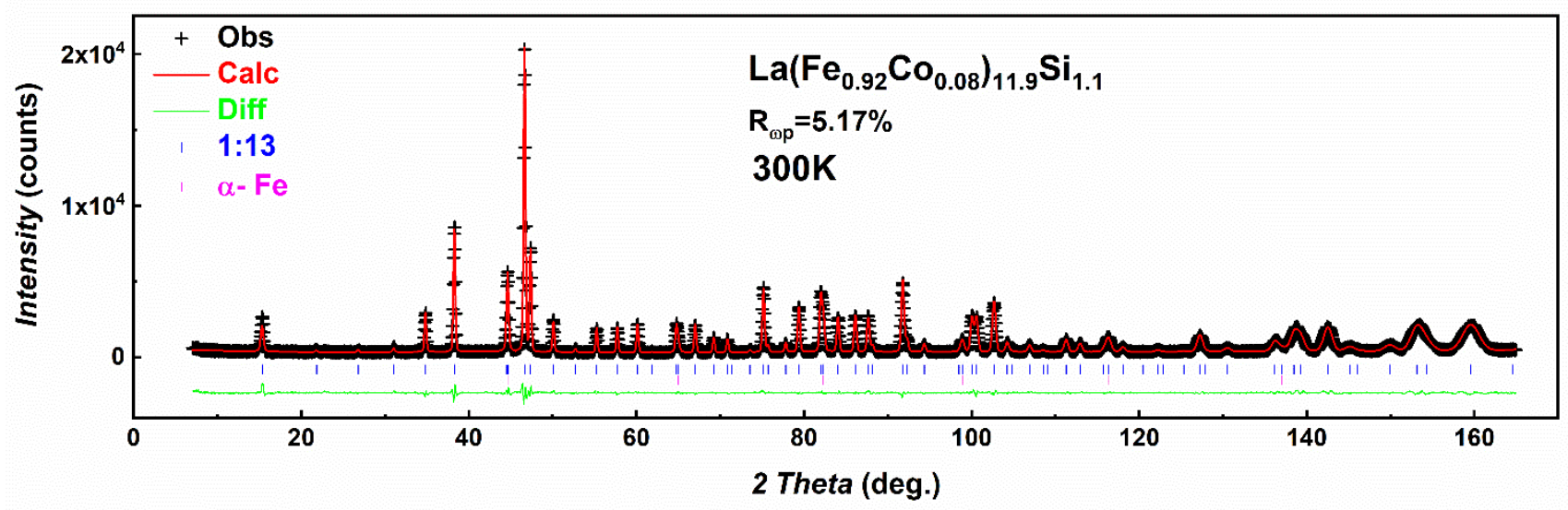

Figure S1. The collected NPD pattern together with the Rietveld refinement plot at $300 \mathrm{~K}$ in the absence of pressure for $\mathrm{La}\left(\mathrm{Fe}_{0.92} \mathrm{Co}_{0.08}\right)_{11.9} \mathrm{Si}_{1.1}$ compound. The black cross and red line represent observed and calculated profiles, respectively. The vertical bars indicate the Bragg reflection positions of $\mathrm{NaZn}_{13}$-phase (blue) and $\alpha$-Fe (purple). 


\section{Crystal structure parameters of $\mathrm{La}\left(\mathrm{Fe}_{0.92} \mathrm{Co0.08}\right)_{11.9} \mathrm{Si}_{1.1}$ compound under different pressures}

\section{obtained from the refinements of power neutron diffraction data.}

Table S1. Crystal structure parameters of ferromagnetic (FM) and paramagnetic (PM) phase of $\mathrm{La}\left(\mathrm{Fe}_{0.92} \mathrm{Co}_{0.08}\right)_{11.9} \mathrm{Si}_{1.1}$ compound under different pressures obtained from the refinements of power neutron diffraction data: the lattice constant a, unit cell volume $V$, variation of lattice parameters and unit cell volumes across phase transition, the fractional coordinates at $96 \mathrm{i}$ site y, z, La-Fe 2 atomic distance, intra-icosahedral $\mathrm{Fe}-\mathrm{Fe}$ distances (B1, B2, B3) and inter-icosahedral ones(B4, B5), angles of Fe-Fe-Fe atomic chain (intra-icosahedron: Ang-1', Ang-2', Ang-3', Ang-4', on surface of the icosahedron (Ang-1, Ang-2), inter-icosahedron (Ang-3, Ang-4, Ang-5, Ang-6, Ang-7), Figure 4b), and the reliability factors $R_{\omega p}$.

\begin{tabular}{|c|c|c|c|c|c|c|c|c|c|c|c|c|}
\hline & \multicolumn{3}{|c|}{ 0kbar } & \multicolumn{3}{|c|}{$3 \mathrm{kbar}$} & \multicolumn{3}{|c|}{$6 \mathrm{kbar}$} & \multicolumn{3}{|c|}{ 9kbar } \\
\hline & $\begin{array}{c}\text { FM } \\
(242 K)\end{array}$ & $\begin{array}{c}\text { PM } \\
(338 K)\end{array}$ & $\begin{array}{l}\text { Diff } \\
(\%)\end{array}$ & $\begin{array}{c}\text { FM } \\
(201 K)\end{array}$ & $\begin{array}{c}\text { PM } \\
(314 K)\end{array}$ & $\begin{array}{l}\text { Diff } \\
(\%) \\
\end{array}$ & $\begin{array}{c}\text { FM } \\
(176 K)\end{array}$ & $\begin{array}{c}\text { PM } \\
(302 K)\end{array}$ & $\begin{array}{l}\text { Diff } \\
(\%)\end{array}$ & $\begin{array}{c}\text { FM } \\
(149 K)\end{array}$ & $\begin{array}{c}\text { PM } \\
(284 \mathrm{~K}) \\
\end{array}$ & $\begin{array}{l}\text { Diff } \\
(\%) \\
\end{array}$ \\
\hline $\mathbf{a}(\AA)$ & $11.5333(3)$ & $11.4908(3)$ & -0.37 & 11.5237 (3) & $11.4745(3)$ & -0.43 & $11.5132(3)$ & $11.4564(3)$ & -0.50 & $11.5052(3)$ & 11.4457 (4) & -0.52 \\
\hline V(̊̊3) & $1533.6(2)$ & $1517.2(1)$ & -1.08 & $1530.3(1)$ & $1510.8(1)$ & -1.29 & $1526.1(1)$ & $1503.6(1)$ & -1.50 & $1522.9(1)$ & $1499.4(1)$ & -1.57 \\
\hline y/a & $0.1823(3)$ & $0.1806(3)$ & 0.94 & $0.1822(3)$ & 0.1801 (3) & -1.22 & $0.1816(3)$ & $0.1793(3)$ & -1.28 & 0.1809 (3) & $0.1794(3)$ & 0.82 \\
\hline $\mathbf{z} / \mathbf{a}$ & 0.1198 (3) & 0.1193 (3) & -0.45 & 0.1198 (3) & 0.1195 (3) & 0.27 & 0.1193 (3) & $0.1186(3)$ & -0.60 & $0.1201(3)$ & 0.1187 (3) & -1.15 \\
\hline $\mathrm{La}^{-\mathrm{Fe}_{2}}(\stackrel{\AA}{\mathbf{A}})$ & $3.3431(18)$ & $3.3382(18)$ & -0.15 & $3.3406(18)$ & $3.3339(18)$ & -0.2 & $3.342(18)$ & $3.3355(18)$ & -0.19 & $3.3375(18)$ & $3.3276(18)$ & 0.30 \\
\hline B1(令) & $2.517(3)$ & $2.487(3)$ & -1.21 & $2.514(3)$ & $2.48(3)$ & -1.37 & $2.502(3)$ & $2.463(3)$ & -1.58 & $2.501(3)$ & $2.459(3)$ & -1.71 \\
\hline B2(§̊) & $2.775(5)$ & $2.742(5)$ & -1.20 & $2.766(5)$ & $2.729(5)$ & -1.36 & $2.758(5)$ & $2.721(5)$ & -1.36 & $2.755(5)$ & $2.712(5)$ & -1.59 \\
\hline B3(@̊) & $2.618(3)$ & $2.585(3)$ & -1.28 & $2.615(3)$ & $2.576(3)$ & -1.51 & $2.602(3)$ & $2.563(3)$ & -1.52 & $2.599(3)$ & $2.556(3)$ & -1.68 \\
\hline 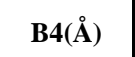 & $2.384(5)$ & $2.405(5)$ & 0.87 & $2.392(5)$ & $2.407(5)$ & 0.62 & $2.393(5)$ & $2.415(5)$ & 0.91 & $2.394(5)$ & $2.413(5)$ & 0.79 \\
\hline B5( $(̊)$ & $2.500(4)$ & $2.510(4)$ & 0.40 & $2.499(4)$ & $2.517(4)$ & 0.72 & $2.504(4)$ & $2.513(4)$ & 0.36 & $2.511(4)$ & $2.519(4)$ & 0.32 \\
\hline Ang-1' $\left({ }^{\circ}\right)$ & $66.72(17)$ & $67.16(17)$ & 0.66 & $66.62(17)$ & $66.69(17)$ & 0.11 & $66.60(17)$ & $66.97(17)$ & 0.56 & $67.17(17)$ & $66.98(17)$ & -0.28 \\
\hline Ang-2, $\left({ }^{\circ}\right)$ & $56.66(15)$ & $56.42(15)$ & 0.42 & $56.69(15)$ & $56.66(15)$ & -0.05 & $56.70(15)$ & $56.52(15)$ & -0.32 & $56.42(15)$ & $56.51(15)$ & 0.16 \\
\hline Ang-3' $\left({ }^{\circ}\right)$ & $62.66(11)$ & $62.56(11)$ & 0.16 & $62.68(11)$ & $62.67(11)$ & -0.02 & $62.68(11)$ & $62.60(11)$ & 0.13 & $62.56(11)$ & $62.60(11)$ & 0.06 \\
\hline Ang-4' $\left({ }^{\circ}\right)$ & $58.69(11)$ & $58.72(11)$ & 0.05 & $58.66(11)$ & $58.67(11)$ & 0.02 & $58.66(11)$ & $58.70(11)$ & 0.07 & $58.72(11)$ & $58.70(11)$ & -0.03 \\
\hline Ang-1 $\left({ }^{\circ}\right)$ & $58.07(13)$ & $57.82(13)$ & 0.43 & $58.13(13)$ & $58.09(13)$ & -0.07 & $58.14(13)$ & $57.93(13)$ & -0.36 & $57.82(13)$ & $57.92(13)$ & 0.17 \\
\hline Ang-2 $\left(^{\circ}\right)$ & $63.85(15)$ & $64.36(15)$ & 0.80 & $63.73(15)$ & $63.81(15)$ & 0.13 & $63.71(15)$ & $64.14(15)$ & 0.67 & $64.37(15)$ & $64.15(15)$ & 0.34 \\
\hline Ang-3 $\left(^{\circ}\right)$ & $56.48(16)$ & $56.92(16)$ & 0.78 & $56.47(16)$ & $56.93(16)$ & 0.81 & $56.68(16)$ & $57.27(16)$ & 1.04 & $56.74(16)$ & $57.24(16)$ & 0.88 \\
\hline Ang-4 $\left(^{\circ}\right)$ & $67.03(18)$ & $66.17(18)$ & -1.28 & $67.06(18)$ & $66.14(18)$ & -1.37 & $66.63(18)$ & $65.45(18)$ & -1.77 & $66.53(18)$ & $65.52(18)$ & -1.52 \\
\hline Ang-5 $\left(^{\circ}\right)$ & $55.66(15)$ & $56.15(15)$ & 0.88 & $55.71(15)$ & $56.50(15)$ & 1.42 & $56.11(15)$ & $56.92(15)$ & 1.44 & $55.81(15)$ & $56.85(15)$ & 1.86 \\
\hline Ang-6 $\left({ }^{\circ}\right)$ & $64.52(16)$ & $63.25(16)$ & -1.97 & $64.60(16)$ & $63.45(16)$ & -1.78 & $64.08(16)$ & $62.50(16)$ & -2.47 & $63.68(16)$ & $62.57(16)$ & -1.74 \\
\hline Ang-7 $\left({ }^{\circ}\right)$ & $59.82(16)$ & $60.60(16)$ & 1.30 & $59.70(16)$ & $60.05(16)$ & 0.59 & $59.8(16)$ & $60.58(16)$ & 1.30 & $60.51(16)$ & $60.58(16)$ & 0.12 \\
\hline $\mathbf{R}_{\omega \mathrm{p}}(\%)$ & 6.78 & 6.44 & - & 7.29 & 6.49 & - & 6.98 & 5.99 & - & 6.63 & 6.85 & - \\
\hline
\end{tabular}




\section{The total and partial electronic DOS of up spins and down spins for $\mathrm{La}\left(\mathrm{Fe}_{0.92} \mathrm{Co0.08}_{11.9} \mathrm{Si}_{1.1}\right.$ compound in the ferromagnetic state at ambient pressure.}

It is worth noting that a very denser energy point is required to describe the DOSs near the Fermi level for such large and complicated magnetic compound. All of the DOSs reported in this work are plotted with an energy-range from $-8 \mathrm{eV}$ to $4 \mathrm{eV}$ with 12000 energy points (NEDOS=12000) and a small broadening energy of $0.01 \mathrm{eV}(\mathrm{SIGMA}=0.01)$.

To understand the mechanism of magnetic behavior of $\mathrm{La}\left(\mathrm{Fe}_{0.92} \mathrm{Co}_{0.08}\right)_{11.9} \mathrm{Si}_{1.1}$, we have calculated the total and partial electronic density of states (DOS), as shown in Figure S2. The Fermi energy is shifted to zero $\left(E_{F}=0\right)$. There is a large splitting between the spin-up and spin-down DOS below the $E_{F}$, as shown in Figure S2a, which result a large magnetic moment of $210.16 \mu_{\mathrm{B}}$ at ambient pressure. The spin-up bands is almost completely filled, while the spin-down bands is partially filled with some spin-down holes, thus using the familiar terminology, we can consider the $\mathrm{La}\left(\mathrm{Fe}_{0.92} \mathrm{Co}_{0.08}\right)_{11.9} \mathrm{Si}_{1.1}$ compound as strong ferromagnetism. The spin-up and spin-down DOS peaks from $-5 \mathrm{eV}$ to $0 \mathrm{eV}$ are mainly from the $3 \mathrm{~d}$ bands of Fe and Co atoms (see Figure S2c and Figure S2d), and the splittings result a local magnetic moment of $1.91 \sim 2.56 \mu_{\mathrm{B}}$ on the Fe sites and $1.41 \sim 1.65 \mu_{\mathrm{B}}$ on the Co sites, respectively. There is a small induced splitting of $5 \mathrm{~d}$ bands of La around the energy of $-1.5 \mathrm{eV}$ (which induce a small magnetic moment of -0.21 $\mu_{\mathrm{B}}$ on La sites), indicating the La-5d and (Fe,Co)-3d bands are well hybridized, but the main part of the partial DOS of the La-5d is located above the Fermi level (Figure S2b). Also there is a small induced splitting of $2 \mathrm{p}$ bands of $\mathrm{Si}$ around the energy range from $-6.0 \mathrm{eV}$ to $-1.0 \mathrm{eV}$, as shown in Figure S2e, which induce a small magnetic moment of $-0.11 \mu_{\mathrm{B}}$ on Si sites. Meanwhile the peaks from the $3 \mathrm{~s}$ bands of Si are located around the energy of $-8 \mathrm{eV}$ (Figure S2e), lying far below $E_{F}$ and the peaks from the $4 \mathrm{f}$ bands of La are around the energy of $3 \mathrm{eV}$, above $E_{F}$ (Figure S2b). 


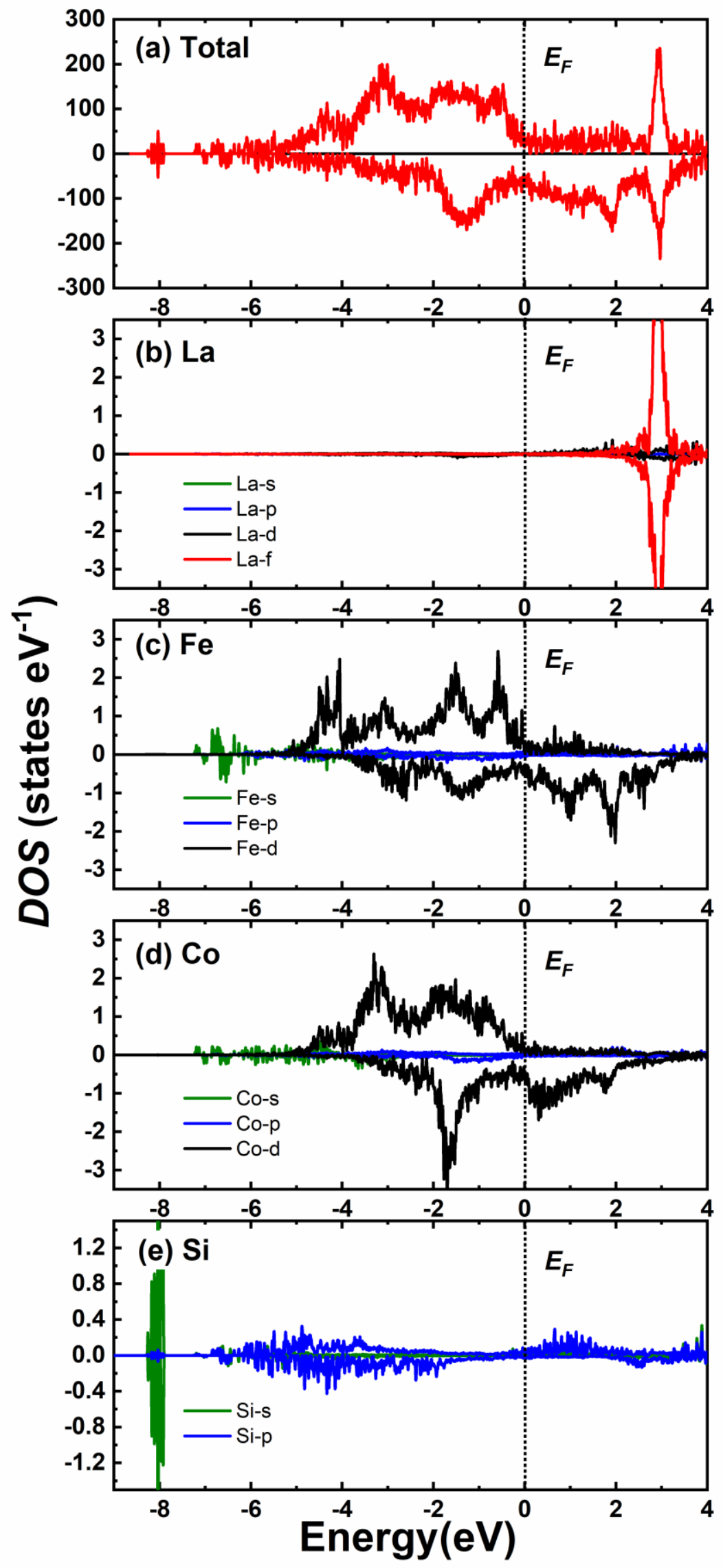

Figure S2. The total and partial electronic DOS of up spins and down spins for $\mathrm{La}\left(\mathrm{Fe}_{0.92} \mathrm{Co}_{0.08}\right)_{11.9} \mathrm{Si}_{1.1}$ compound in the ferromagnetic state at ambient pressure. The Fermi energy is shifted to zero. 


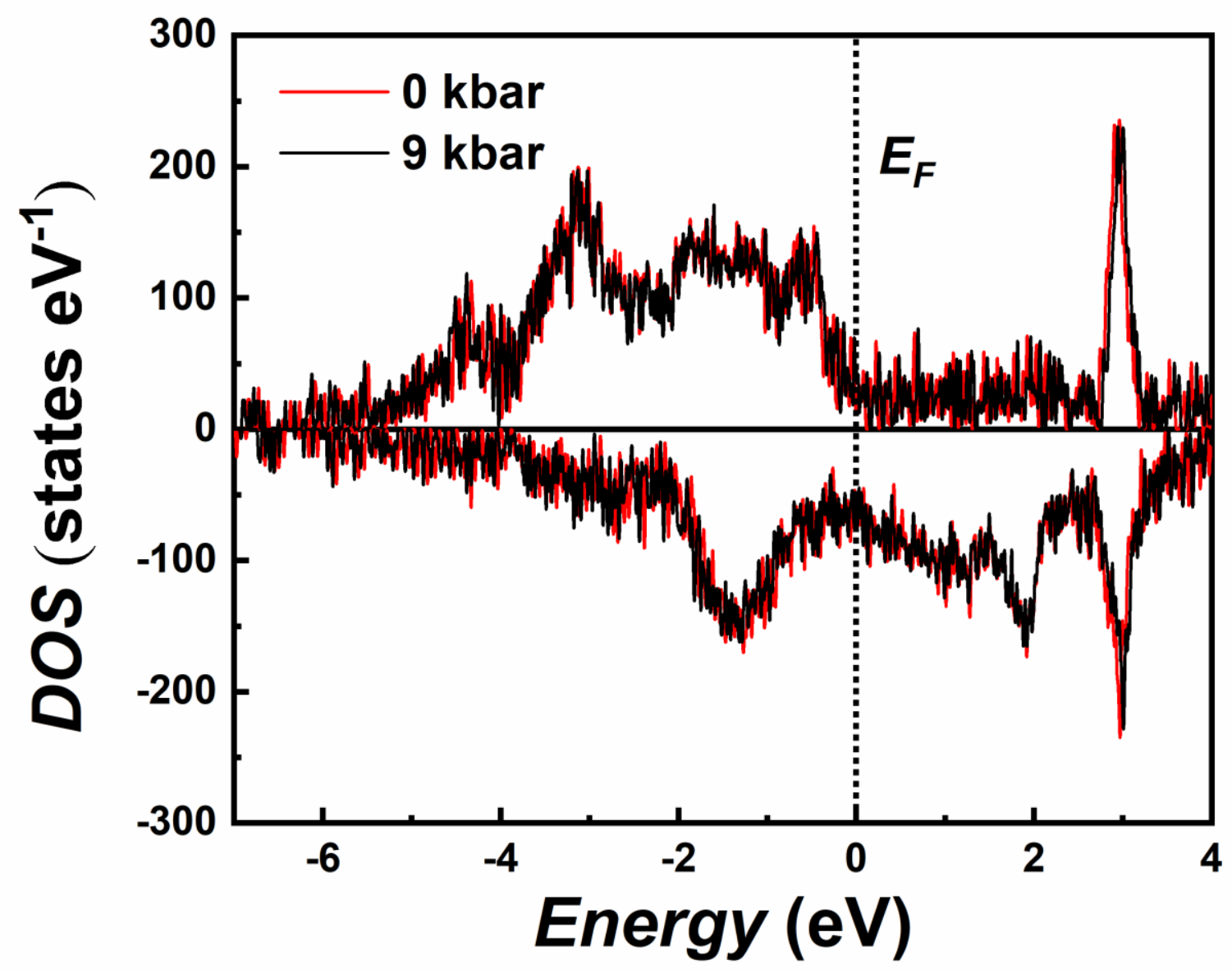

Figure S3. The total density of states of $\mathrm{La}\left(\mathrm{Fe}_{0.92} \mathrm{Co}_{0.08}\right)_{11.9} \mathrm{Si}_{1.1}$ compound in the ferromagnetic state under pressures 0 and 9kbar. The Fermi energy is shifted to zero.

\section{Evaluation of the enlarged $\Delta V / V$ to the enhancement of BCE by hydrostatic pressure.}

The barocaloric effect (BCE) of $\mathrm{La}\left(\mathrm{Fe}_{0.92} \mathrm{Co}_{0.08}\right)_{11.9} \mathrm{Si}_{1.1}$ shows a rapid increase with pressure due to the enhanced magnetovolume effect and the evolution of the transition nature by hydrostatic pressure. To understand the essence, we quantitatively evaluate the contribution of enlarged volume change $\Delta V / V$ during phase transition to the enhancement of BCE. Firstly, we assume that the effect of pressure on the movement and evolution of phase transition order remains unchanged but does not affect the $\Delta V / V$. Then the lattice entropy $S_{\text {Latt-PT }}$ involving phase transition under different pressures can be calculated based on Debye approximation, as shown in Figure S4a. By comparing the $S_{\text {Latt-PT }}$ with and without pressure, we can get the pressure-induced lattice entropy change $\Delta S_{\text {Latt }}$, i.e. the contribution of lattice entropy to the barocaloric effect $\left(\Delta S_{B C E-L a t t}{ }^{\prime}\right.$, as plotted in Figure S4b. We also suppose that the spin contribution to the 
$\mathrm{S}_{\text {Tot-PT }}$ remains nearly independent of pressure (Figure 7d). Then total entropy $S^{\prime}{ }_{T o t-P T}$ involving phase transition (Figure S4c), as well as the pressure-induced total entropy change $\Delta S_{B C E-T o t}^{\prime}($ Figure S4d), i.e. the barocaloric effect (BCE') without considering the effect of enlarged $\Delta V / V$, can be obtained. Figure $7 \mathrm{~g}$ and Figure $7 \mathrm{~h}$ depict the comparison between $\Delta S_{B C E \text {-Latt }}^{\prime}$ and $\Delta S_{B C E-L a t t}$, and between $\Delta S^{\prime}{ }_{B C E-T o t}$ and $\Delta S_{B C E-T o t}$, respectively, where the shaded area denotes the contribution from enlarged $\Delta V / V$ under $9 \mathrm{kbar}$.

One can note that, compared to $\Delta S_{B C E-L a t t}$ and $\Delta S_{B C E-T o t}{ }_{B}$, the maximum of $\Delta S_{B C E-L a t t}$ and $\Delta S_{B C E-T o t}$ curves increases by $39 \%$ and $22 \%$, while the area under the curves which connects RC enlarges by $47 \%$ and $20 \%$, respectively, due to the contribution of enlarged $\Delta V / V$ by hydrostatic pressure.

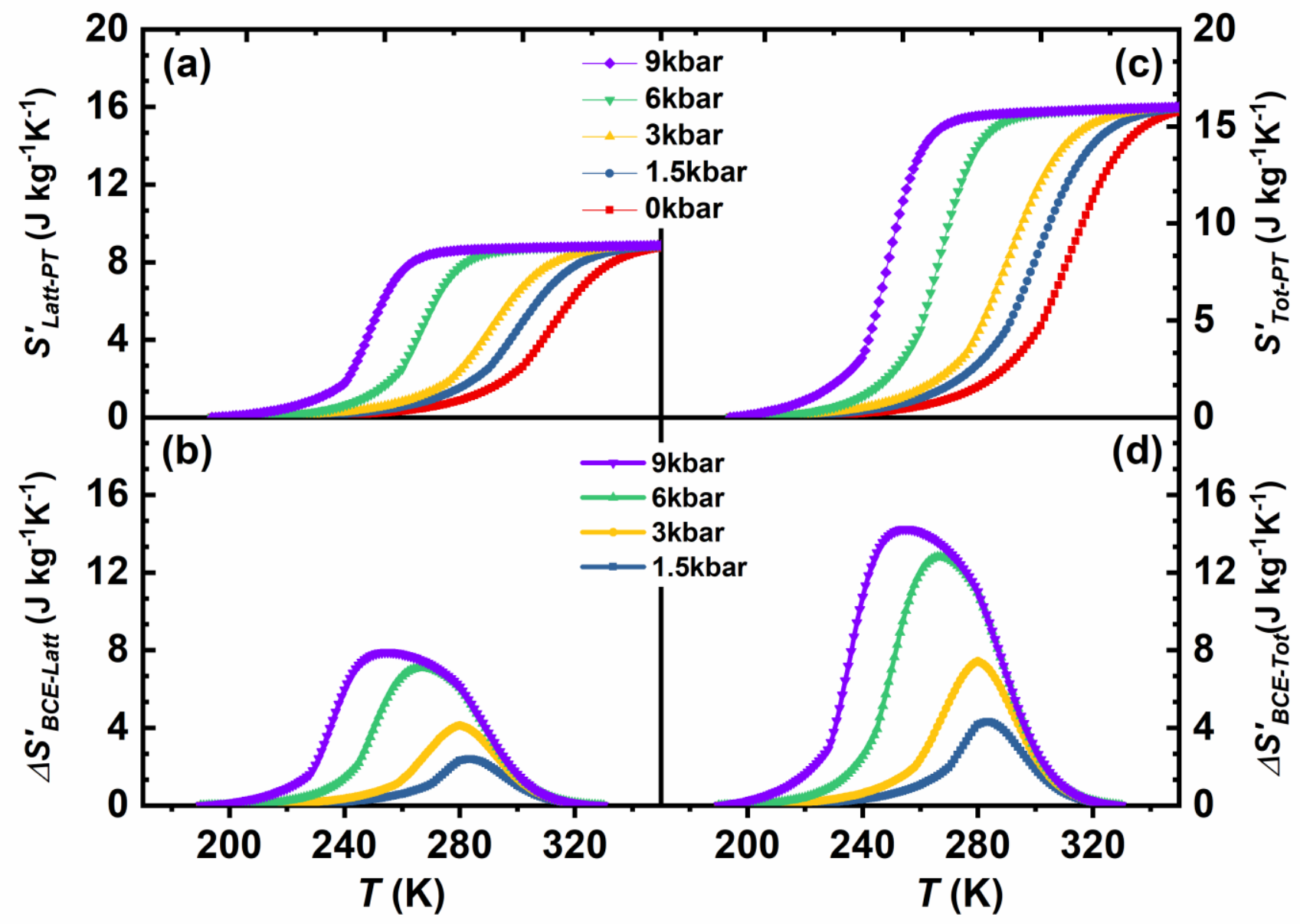

Figure S4. Supposing that the effect of pressure on the movement and evolution of phase transition order remains unchanged but does not affect the $\Delta V / V$, (a) the calculated lattice entropy $S^{\prime}{ }_{L a t t-P T}$ involving phase transition based on Debye approximation, (b) pressure-induced lattice entropy change $\Delta S_{B C E \text {-Latt, }}$, i.e. the lattice contribution to barocaloric effect $\left(\triangle S_{B C E-L a t t}^{\prime}\right)$, (c) the total entropy $S^{\prime}{ }_{T o t-P T}$ involving phase transition only under different pressures, and (d) the pressure-induced total entropy change $\Delta S^{\prime}{ }_{B C E-T o t}$, i.e. the barocaloric effect (BCE') without considering the effect of enlarged $\Delta V / V$.

IV. Debye temperature $\Theta_{D}$ of $\mathrm{La}\left(\mathrm{Fe}_{0.92} \mathrm{CoO}_{0.08}\right)_{11.9} \mathrm{Si}_{1.1}$ in the absence of pressure. 


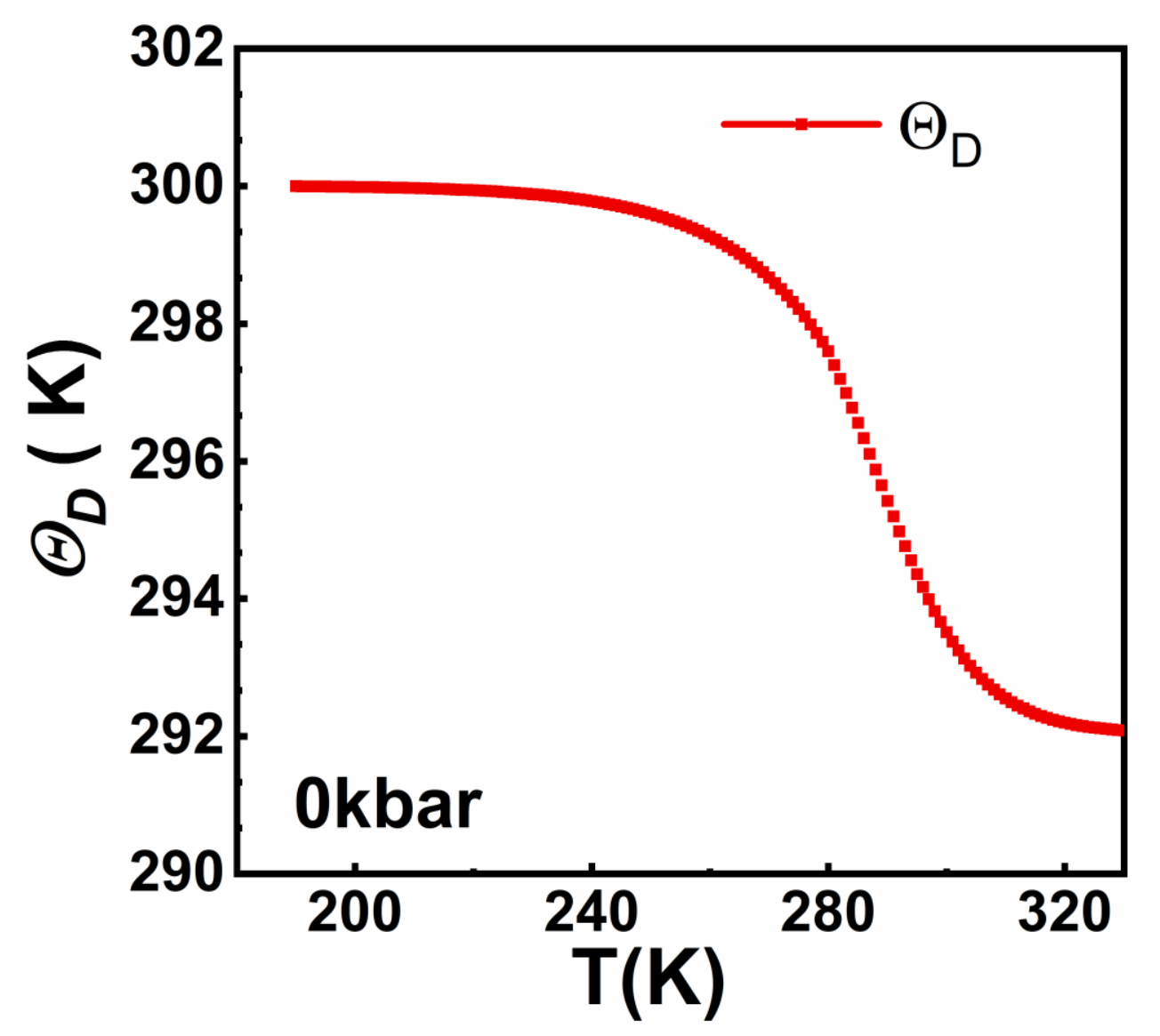

Figure S5 Temperature dependence of $\Theta_{\mathrm{D}}$ for $\mathrm{La}\left(\mathrm{Fe}_{0.92} \mathrm{Co}_{0.08}\right)_{11.9} \mathrm{Si}_{1.1}$ calculated by Debye approximation in the absence of pressure. 